\title{
REVIEW OF VARIOUS ADAPTIVE MODULATION AND CODING TECHNIQUES IN WIRELESS NETWORK
}

\author{
Lily Mishra ${ }^{1}$, M.H.Patwardhan ${ }^{2}$ \\ ${ }^{1,2}$ Department of Electronics and Telecommunication, D. J. Sanghvi college of Engineering, Mumbai
}

\begin{abstract}
Adaptive modulation and coding (AMC) is a method which adapts its transmitting parameters according to the channel state and is used in various modern wireless communications to maximize spectrum efficiency by minimizing the error rate. One of the driving strengths of AMC is the Signal-to-Noise Ratio (SNR) estimation and feedback channel for adaptation. Degrading effects caused due to sudden variation in the channel over time sometimes require the transmission link to react appropriately so it can minimize the Bit Error Rate (BER). There are various adaptive methods to implement the same like variable rate, variable error probability, and variable coding or hybrid technique. This article focuses upon variable power technique and describes two to three different power techniques and tries to make a comparison between two of them i.e, channel inversion and water-filling.
\end{abstract}

Keywords- Adaptive Modulation and Coding (AMC), Bit Error Rate (BER), channel inversion, water-filling $* * *$

\section{INTRODUCTION}

The ever increasing demand of all types of services, voice, data and above all, multimedia services, requires the design of increasingly more intelligent and nimble communication systems, which are capable of providing access to spectrally efficient and flexible data rate. These systems are able to adapt and adjust the transmission parameters based on the link quality, hence improving the spectrum efficiency of the system, and in this way, reaching the capacity limits of the underlying wireless channel. Link adaptation techniques, often referred to as adaptive modulation and coding (AMC), are a good way for reaching the cited requirements. They are so designed to track the channel variations, thus changing the modulation and coding scheme to yield a higher throughput by transmitting with high information rates under favourable channel conditions and reducing the information rate in response to degradation effects of the channel. In 1968 Hayes suggested an "Adaptive Feedback Communications" system to combat the variations introduced by a MFC (multipath fading channel) with a Rayleigh distributed envelope. The technique was based on some parameter(s) in the communication system being dependent on the state of the channel. In 1972 another approach to adjust the data rate of the communication system was proposed by Cavers. The channel capacity or the maximum average spectral efficiency (MASE), for a communication system utilizing perfect channel state information (CSI) was presented by Goldsmith and Varaiya, in 1997. It was also shown that a variable-rate and constant power scheme has a performance close to the capacity under the same idealized conditions. In [Chua and Goldsmith, 1997; Goldsmith and Chua, 1997] adaptive modulation was combined with channel coding leading to so-called ACM. In paper [2], AMC has been used as an abbreviation for adaptive modulation and coding and 7 different MCS (modulation and coding scheme) levels have been taken in a HSDPA environment and it is shown how the channel toggles between all of them as per the channel condition and trying to achieve the required target of SNR. Thus altogether a comparison between all of them is presented in a simplified manner.

In paper [3], three different processes involved in communication over a wireless channel have been discussed and link adaptation is one of them. Here, link adaptation has been broken into two parts-power control and rate control and rate control has been given the name adaptive modulation and coding using the water-filling concept.

\section{MATERIALS AND METHODS}

There are basically five different types of adaptive methods based upon the parameter that might be controlled for variation. They are:-

\subsection{Variable-Rate Techniques}

- Data rate $\mathrm{R}(\Upsilon)$ is varied as per channel gain $\Upsilon$

- Fix symbol rate $R_{S}=1 / T_{S}$ and use multiple modulation schemes or increase constellation size.

- Fix modulation but change symbol rate

\subsection{Variable-Power Techniques}

- Transmit power is changed to compensate for SNR variations due to fading. 


\subsection{Variable Error Probability}

- Instantaneous BER is adapted subject to an average BER constraint $\overline{P b}$ where

$$
\overline{P_{b}}=\int P_{b}(\gamma) p(\gamma) d \gamma
$$

\subsection{Variable Coding Techniques}

- $\quad$ Stronger error correction code when $\Upsilon$ is small

- Weaker error correction code when $\Upsilon$ is large

\subsection{Hybrid Techniques}

- Joint optimization of different techniques to meet a given performance requirement

Out of all the above methods, this article particularly focuses upon bringing out a comparison between two important types of power variable techniques i.e., channel inversion and waterfilling method.

Both the techniques are briefly discussed below:-

\subsection{Channel Inversion}

Suboptimal transmitter adaptation scheme

- Transmitter uses CSI to maintain a constant received power(hence channel fading is inverted)

- Given by $P(\Upsilon) / P=\sigma / \Upsilon$, where $\sigma=$ const. received SNR with transmit power constraint

$$
\int_{0}^{\infty} P(\gamma) p(\gamma) d \gamma \leq \bar{P}
$$

Hence, $\sigma=1 / \mathrm{E}[1 / \Upsilon]$

\subsection{Water-Filling}

It represents same power adaptation policy that achieves capacity with $\Upsilon$ replaced with $\Upsilon_{k}$.

$$
\begin{aligned}
& \frac{P(\gamma)}{\bar{P}}=\left\{\begin{array}{cc}
\frac{1}{\gamma_{0}}-\frac{1}{\gamma K} & \gamma \geq \frac{\gamma_{0}}{K}=\gamma_{K} \\
0 & \gamma<\frac{\gamma_{0}}{K}=\gamma_{K}
\end{array}\right. \\
& \frac{K P(\gamma)}{\bar{P}}=\left\{\begin{array}{cc}
\frac{1}{\gamma_{K}}-\frac{1}{\gamma} & \gamma \geq \gamma_{K} \\
0 & \gamma<\gamma_{K}
\end{array}\right.
\end{aligned}
$$

Other types of power variable technique include truncated channel inversion which is same as channel inversion except that in this method those channels are simply discarded which does not fulfill a minimum threshold value of SNR. And, another is mercury-filling dicussed in [15] where power is allocated first to the channel having the highest gain and then rest of the power is allocated in a descending order of gain to other channels. The basic block diagram that is used to implement any adaptive and modulation technique is as follows:-

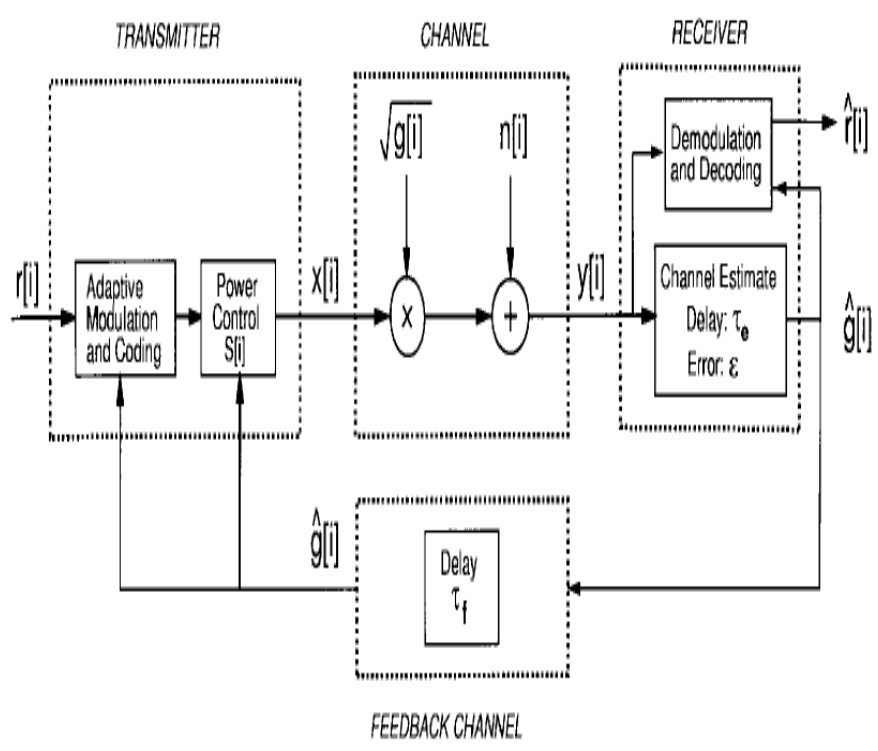

Where a discrete-time channel with stationary and ergodic time varying gain $V_{\mathrm{g}[\mathrm{i}]} ; 0 \leq \mathrm{g}[\mathrm{i}]$, and $\mathrm{AWGN} \mathrm{n}[\mathrm{i}]$ is considered. It is assumed that the channel power gain $\mathrm{g}[\mathrm{i}]$ is independent of the channel input and has an expected value as unity. Let $\mathrm{S}$ denote the average transmit signal power, N0 denote the noise density of $n[i]$, and $B$ denote the bandwidth of the received signal. The instantaneous received signal-to-noise ratio $(\mathrm{SNR})$ is then $[\mathrm{i}]=\mathrm{Sg}[\mathrm{i}] /(\mathrm{N} 0 \mathrm{~B})$, and its expected value overall time is $\mathrm{S} /(\mathrm{N} O \mathrm{~B})$.

The system model, which sends an input message w from the transmitter to the receiver, is illustrated in the figure below. The message is encoded into the codeword $\mathrm{x}$, and is transmitted over the time varying channel as $x[i]$ at time $i$. The channel gain $\mathrm{g}[\mathrm{i}]$ changes over the transmission of the codeword. Perfect instantaneous channel estimation is assumed so that the channel power gain $\mathrm{g}[\mathrm{i}]$ is known to the receiver at time $\mathrm{i}$. We also consider the case when $\mathrm{g}[\mathrm{i}]$ is known to both the receiver and transmitter at time $i$, as could be obtained through an error-free delay less feedback path. This allows the transmitter to adapt $\mathrm{x}[\mathrm{i}]$ to the channel gain at time $i$, and is a genuine model for a slowly varying channel with channel estimation and transmitter feedback. 


\section{RESULTS AND DISCUSSION}

In the following table all the papers have been categorized on the basis of which context they are used in, what is the target they want to achieve and what is their performance criteria:-

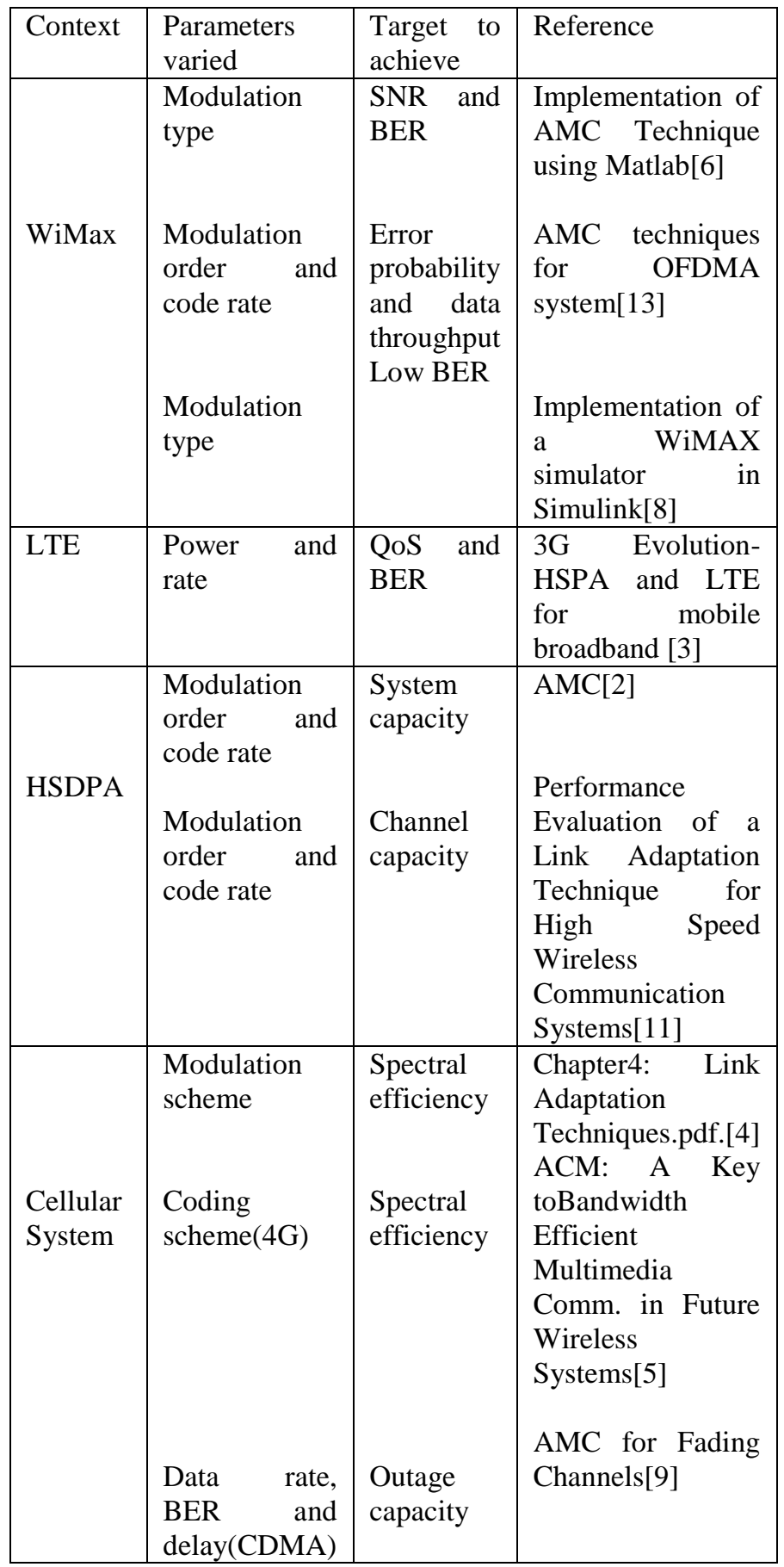

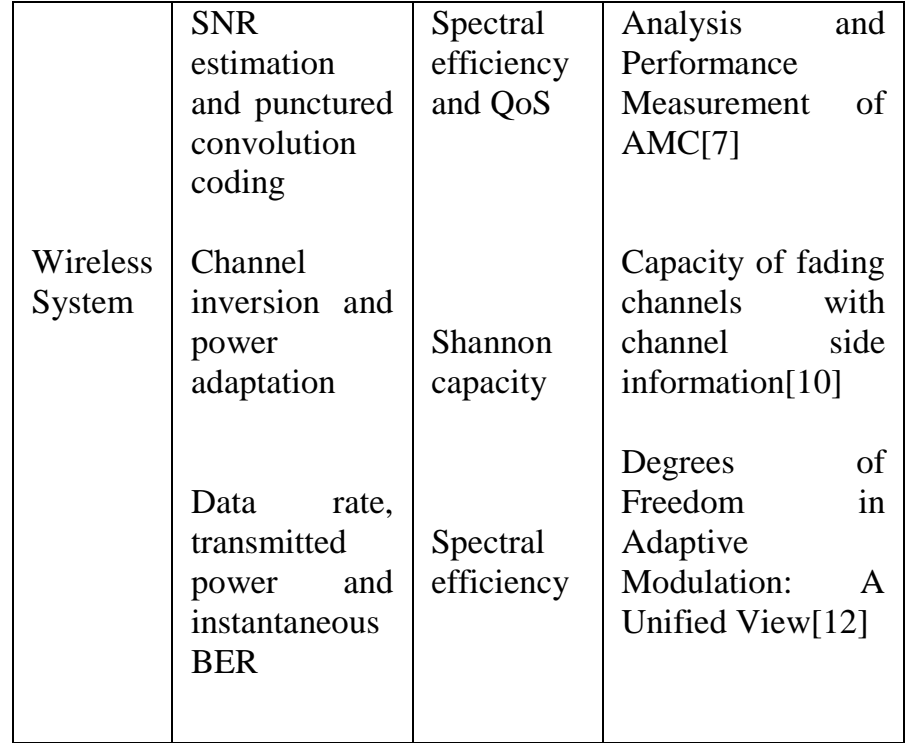

The above table clarifies that adaptive modulation and coding is applicable in different environments like Wi-max, LTE, cellular system and other general wireless systems. With the variation in different parameters different targets like enhanced value of SNR, higher spectral efficiency, more outage capacity and better quality of service may be achieved as per the requirement.

\subsection{Capacity Comparison}

When different power variation techniques are compared capacity-wise(spectral efficiency) in Rayleigh fading channel, at low SNR(Y) values they perform more or less same but as the value of SNR increases, optimal adaptation water-filling technique is observed performing nearer to the AWGN channel capacity, hence the best among channel inversion, inverted channel inversion and water-filling. Water-filling technique is otherwise known as optimal adaptation technique and channel inversion is also known as sub-optimal power adaptation technique. The performance of truncated channel inversion is better than channel inversion but not as good as that of water-filling. One more technique is there where receiver side information is made available to the transmitter's side. In that case whatever performance is seen is same as that of inverted channel inversion. The equations describing the capacity in all the four cases have been given below.

Capacity in case of optimal adaptation(water-filling):-

$$
C(S)=\max _{S(\gamma): \int S(\gamma) p(\gamma)=S} \int_{\gamma} B \log \left(1+\frac{S(\gamma) \gamma}{S}\right) p(\gamma) d \gamma \ldots(1)
$$

Capacity in case of receiver side information:-

$$
C(S)=\int B \log (1+\gamma) p(\gamma) d \gamma \ldots(2)
$$


Capacity in case of truncated channel inversion:-

$$
C(S)=\max _{\gamma_{0}} B \log \left[1+\frac{1}{E_{\gamma_{0}}[1 / \gamma]}\right] p\left(\gamma \geq \gamma_{0}\right) \ldots(3)
$$

Capacity in case of channel inversion:-

$$
C(S)=B \log \left[1+\frac{1}{E[1 / \gamma]}\right] \ldots \text { (4) }
$$

[8] Amalia Roca, "Implementation of a WiMAX simulator in Simulink,"Vienna February 2007.

Where symbols have their usual meanings

[9] Andrea Goldsmith, "Adaptive Modulation and Coding for Fading Channels," 1999 IEEE ITW. Kruger National Park. South Africa. June 20 -25.

[10] Andrea J. Goldsmith and Pravin P. Varaiya, "Capacity of fading channels with channel side information," IEEE TRANSACTIONS ON INFORMATION THEORY, VOL. 43, NO. 6, NOVEMBER 1997.

[11] Lorenzo Caponi,Francesco Chiti, and Romano Fantacci, "Performance Evaluation of a Link Adaptation Technique for High Speed Wireless Communication Systems," IEEE TRANSACTIONS ON WIRELESS COMMUNICATIONS, VOL. 6, NO. 12, DECEMBER 2007.

[12] Seong Taek Chung, and Andrea J. Goldsmith, "Degrees of Freedom in Adaptive Modulation:A Unified View," IEEE TRANSACTIONS ON COMMUNICATIONS, VOL. 49, NO. 9, SEPTEMBER 2001.

[13] Romano Fantacci, Dania Marabissi, Daniele Tarchi, and Ibrahim Habib, "Adaptive Modulation and Coding Techniques for OFDMA Systems," IEEE TRANSACTIONS ON WIRELESS COMMUNICATIONS, VOL. 8, NO. 9, SEPTEMBER 2009.

[14] Andreas Zalonis, Natalia Miliou, Ioannis Dagres, Andreas Polydoros, and Hanna Bogucka, "Trends in Adaptive Modulation and Coding," ADVANCES IN ELECTRONICS AND TELECOMMUNICATIONS, VOL. 1, NO. 1, APRIL 2010.

[15] Weisi Guoy, Siyi Wangx, Xiaoli Chux, "Capacity Expression and Power Allocation for Arbitrary Modulation and Coding Rates," IEEE WIRELESS COMMUNICATIONS AND NETWORKING CONFERENCE (WCNC), 2013.

[16] Andreas Muller,Phillip Frank, "Cooperative Interference Prediction for Enhanced Link Adaptation in the 3GPP LTE Uplink,"

[3] Ruiyuan Tian, "3G Evolution-HSPA and LTE for mobile broadband," Chapter7, 2009.

[4] Chapter4: Link Adaptation Techniques.pdf.

[5] K. J. Hole and G. E. Âien, "Adaptive Coding and Modulation: A Key to Bandwidth-Efficient Multimedia Communications in Future Wireless Systems,"Norwegian University of Science and Technology N-7491 Trondheim, Norway.

[6] Sami H. O. Salih, Mamoun M. A. Suliman, "Implementation of Adaptive Modulation and Coding 